\title{
molecules
}

ISSN 1420-3049

(C) 2007 by MDPI

www.mdpi.org/molecules

Full Paper

\section{Synthesis and In-vitro Antitumor Activity of 1-[3-(Indol-1-yl)prop-1-yn-1-yl]phthalazines and Related Compounds}

\author{
Norbert Haider *, Tamara Kabicher, Johann Käferböck and Angelika Plenk \\ Department of Drug and Natural Product Synthesis, Faculty of Life Sciences, University of Vienna, \\ Althanstraße 14, A-1090 Vienna, Austria
}

* Author to whom correspondence should be addressed; E-mail: norbert.haider@univie.ac.at

Received: 2 August 2007; in revised form: 14 August 2007 / Accepted: 14 August 2007 / Published: 17 August 2007

\begin{abstract}
A series of novel 3-(indol-1-yl)prop-1-yn-1-yl-substituted phthalazines and related azines was prepared via a concise pathway by palladium-catalyzed cross-coupling of appropriate halo-azines and $N$-propargylindoles. Some of the compounds exhibited significant antitumor activity in an in-vitro assay.
\end{abstract}

Keywords: 1-[3-(indol-1-yl)prop-1-yn-1-yl]phthalazines, $N$-propargylindoles, palladiumcatalyzed cross-coupling, Sonogashira reaction, antitumor activity.

\section{Introduction}

During the course of a research program at our department, focusing on the synthesis and antitumor activity of polycyclic hetarenes, especially condensed carbazoles of the ellipticine/olivacine type [1-5] (cf. Figure 1) and polycyclic quinones [6-8], we recently described the preparation of novel pentacyclic ellipticine analogs via a route featuring an intramolecular inverse-electron-demand DielsAlder reaction of indolylpropyl-substituted 1,2-diazines as the key step (Scheme 1) [9]. In a routine invitro screening for cytotoxic activity, not only the target compounds, but also one of the intermediates, namely 1-[3-(indol-1-yl)prop-1-yn-1-yl]phthalazine, showed significant tumor cell-growth inhibition. Therefore, this compound with a 1,3-disubstituted propyne unit as the central element was selected as a new lead structure for further exploratory investigations. Here, we report on the synthesis and the 
results of preliminary in-vitro antitumor tests of a focused compound library featuring the same propyne motif with one electron-rich and one electron-deficient hetarene attached at the terminal carbon atoms.

Figure 1. Structures of the alkaloids, ellipticine and olivacine.<smiles>Cc1c2ccncc2c(C)c2c1[nH]c1ccccc12</smiles>

Ellipticine<smiles>Cc1nccc2c(C)c3[nH]c4ccccc4c3cc12</smiles>

Olivacine

Scheme 1. Synthesis of bridged ellipticine/olivacine analogs [9].

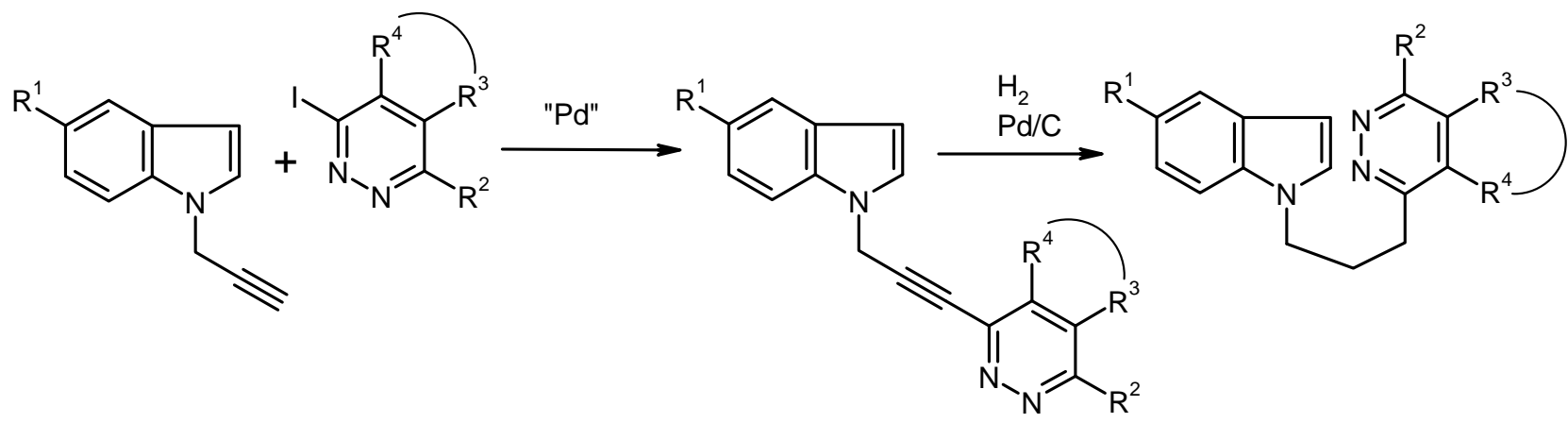

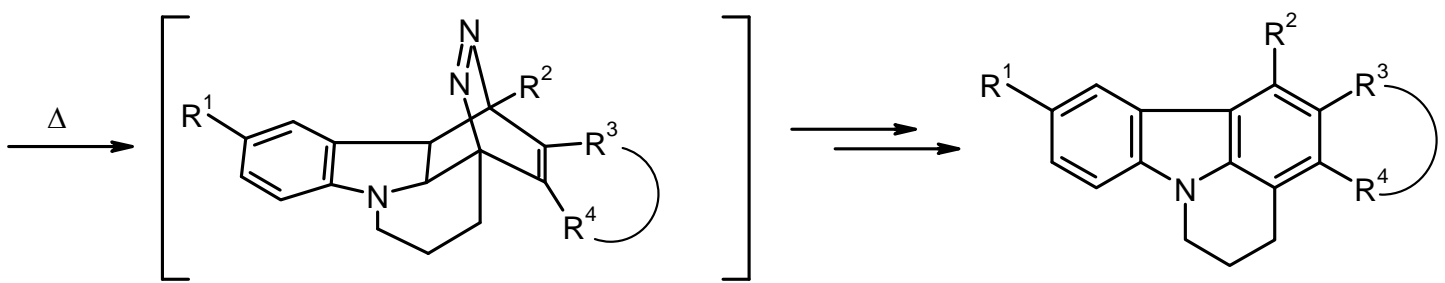

\section{Results and Discussion}

\section{Syntheses}

Following the first step of the pathway above (Scheme 1), the target compounds were prepared essentially by a Sonogashira cross-coupling reaction of an appropriate propargyl-substituted indole or indoline synthon with an iodohetarene or bromohetarene, respectively. Two series of compounds were synthesized, keeping always one of the two heterocyclic subunits constant (either the indole or the azine) and varying the other one. The propynyl-substituted educts were obtained in good yields by 
treatment of the $\mathrm{N}$-unsubstituted precursors with propargyl bromide in toluene/50\% sodium hydroxide, using tetrabutylammonium bromide as a phase-transfer catalyst [10].

\section{An improved synthesis of 1-iodophthalazine}

During the preparation of the requisite starting materials, it turned out that the transformation of 1-chlorophthalazine into 1-iodophthalazine by treatment of the former with potassium iodide and hydroiodic acid in acetone, as described by Hirsch and Orphanos [11], gives very unreliable results if the original work-up procedure is applied (dissolving the initially formed hydroiodide salt of 1-iodophthalazine in water, followed by neutralization and filtration of the free base). In several runs, the compound underwent complete decomposition within a very short time. We found that this crude material is highly acid-sensitive and is prone to an autocatalytic decomposition process if exposed to traces of acid. Therefore, it is essential to keep the $\mathrm{pH}$ after liberation of the free base strictly alkaline. Instead of collecting the product by filtration, it is extracted quickly into dichloromethane and the organic extract is immediately basified by addition of triethylamine (see Experimental section). Evaporation of this solution gives a pure product which can be stored under refrigeration for several weeks.

\section{Palladium-catalyzed cross-coupling reactions}

As the educts for the Sonogashira cross-coupling reaction (see Scheme 2), the following azines were chosen besides 1-iodophthalazine (2a): 1-iodo-4-methylphthalazine (2b) [12], 1-iodo-4-phenylphthalazine (2c) [13], 1-iodoisoquinoline (2d) [14], and 4-bromoisoquinoline (2e) [15]. As the acetylenic building blocks, $N$-propargyl-substituted 5-methoxyindole (1a) [9], 5-bromoindole (1b), methyl indole-5-carboxylate (1c) [16], and indoline (1d) [17] were employed.

Scheme 2. Synthesis of the target compounds (3) by Sonogashira cross-coupling.

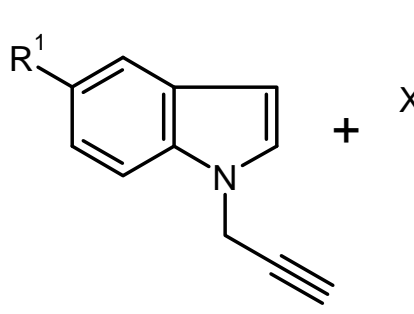

1a-d<smiles>[Y]C1=[Y]=C([R])c2ccccc21</smiles>

2a-e

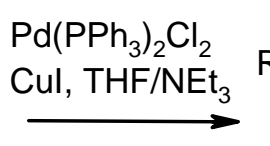<smiles>CCN1CCc2cc([Tl])ccc21</smiles>

3a-g $\mathrm{R}^{1}=\mathrm{H}, \mathrm{OCH}_{3}, \mathrm{Br}, \mathrm{CO}_{2} \mathrm{CH}_{3} \quad \mathrm{R}^{2}=\mathrm{H}, \mathrm{CH}_{3}, \mathrm{Ph} \quad \mathrm{X}=\mathrm{I}, \mathrm{Br} \quad \mathrm{Y}=\mathrm{N}, \mathrm{CH} \quad \mathrm{Z}=\mathrm{N}, \mathrm{CH}$

In all cases, bis(triphenylphosphine)palladium(II)dichloride and copper(I)iodide were used as catalysts, and the reactions were run in tetrahydrofuran/triethylamine under an argon atmosphere at room temperature (except for 3-bromoisoquinoline: reflux temperature) with TLC monitoring. Extended reaction times were found to result in a substantial drop of yields, mainly because of increased decomposition and/or a base-promoted alkyne/allene rearrangement of the products [18] (the latter process was particularly problematic in the case of the 5-bromoindole derivative $\mathbf{3 f}$ ). Whereas 
conversion rates were generally good, yields in some cases were only moderate owing to substantial losses during the purification process. Product structures, reaction conditions and yields of isolated material as well as the results of in-vitro antitumor screening (see below), including the values for the previously prepared analogs $\mathbf{3 h} \mathbf{- j}$ [9] are summarized in Table 1.

Table 1. Structures, reaction conditions, yields, and biological activities for the title compounds.

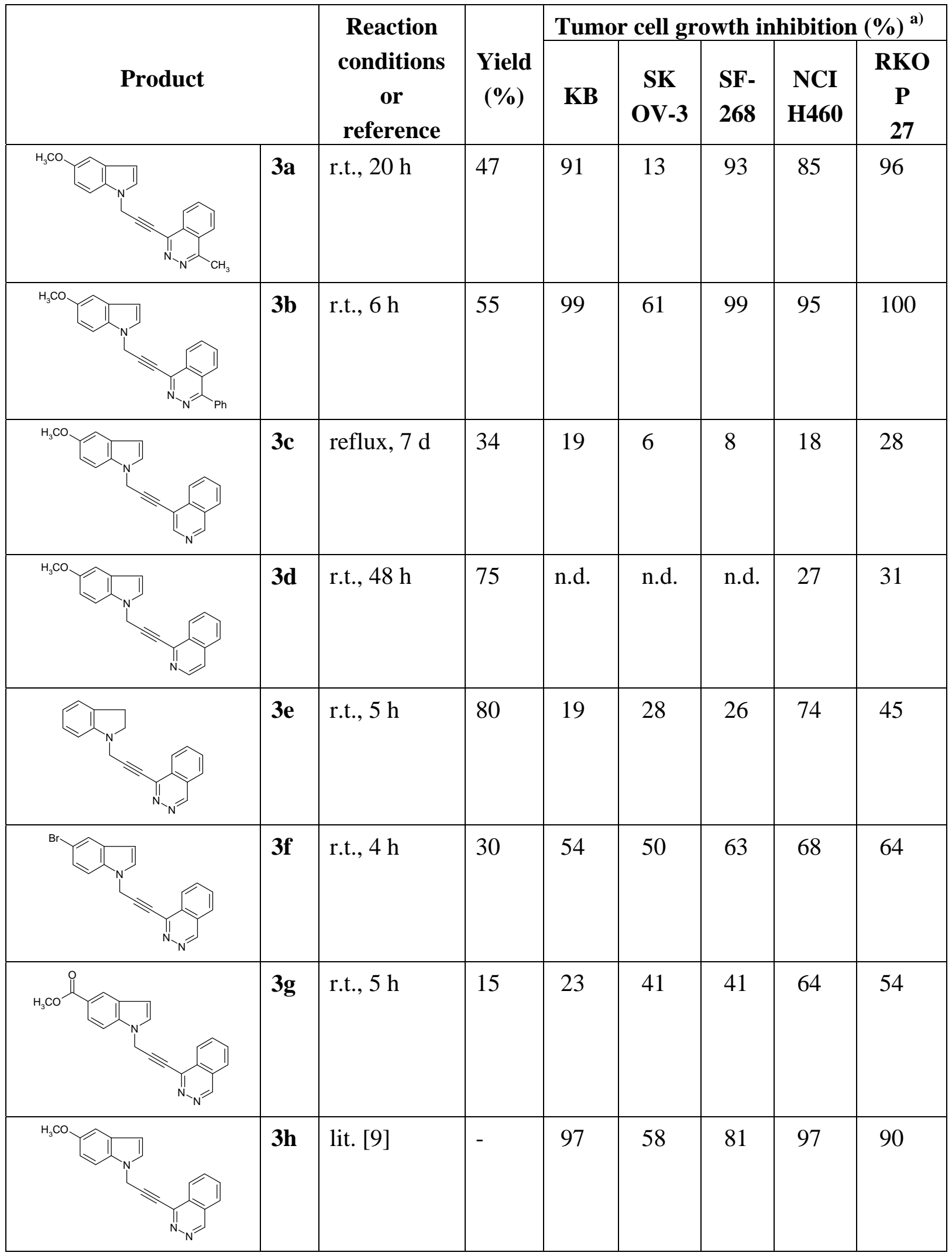


Table 1. Cont.

\begin{tabular}{|l|l|l|l|l|l|l|l|l|}
\hline & $3 \mathbf{i}$ & lit. [9] & - & 28 & 13 & 11 & 36 & 28 \\
\hline & $3 \mathbf{j}$ & lit. [9] & - & 86 & 53 & 88 & 93 & 78 \\
\hline
\end{tabular}

a) Tumor cell lines used: KB: cervical carcinoma; SF-268: CNS cancer; RKOP27: colon adenocarcinoma; SK OV-3: ovarial carcinoma; NCI H-460: non-small-cell lung cancer.

\section{Biological Activity}

All compounds were subjected to a preliminary screening for antitumor activity at a fixed sample concentration of $3.16 \mu \mathrm{g} / \mathrm{mL}$, using the XTT in-vitro assay [19]. As can be concluded from the results (see Table 1), replacement of the phthalazine unit by a monocyclic 1,2-diazine (3i) or by an isoquinoline (3c, 3d) leads to a marked drop in activity, whereas substitution of the phthalazine at position 4 [methyl (3a) or phenyl (3b)] is well tolerated. In the indole part of the molecule, the 5methoxy substituent appears to be most favorable among the variations studied. Replacement of an indole by an indoline structure (3e) results in lower activity.

\section{Conclusions}

Sonogashira reaction of 1-iodophthalazines and related haloazines with the terminal acetylene unit of $\mathrm{N}$-propargylindoles provides a convenient access to the title compounds, which are of pharmaceutical interest due to their activity in an in-vitro antitumor screen. Further investigations will be required for a more detailed evaluation of these agents.

\section{Experimental}

\section{General}

Melting points (uncorrected) were determined on a Kofler hot-stage microscope (Reichert). ${ }^{1} \mathrm{H}-\mathrm{NMR}$ spectra were recorded on a Bruker Avance DPX 200 (200 MHz) or on a Varian UnityPlus 300 (300 MHz) spectrometer. IR spectra were taken on a Perkin-Elmer 1605 FT-IR instrument. Mass spectra were obtained on a Shimadzu QP5050A DI 50 instrument, high-resolution mass spectra were recorded on a Finnigan MAT 8230 spectrometer at the Institute of Organic Chemistry, University of Vienna. Column chromatography was carried out on Merck Kieselgel 60, 0.063-0.200 mm, thin layer chromatography was done on Merck aluminium sheets pre-coated with Kieselgel $F_{254}$. Microanalyses were performed at the Microanalytical Laboratory, Faculty of Chemistry, University of Vienna. 
5-Bromo-1-prop-2-yn-1-yl-1H-indole (1c). To a solution of 5-bromoindole (1.96 g, $10 \mathrm{mmol})$ and propargyl bromide (2.23 g of a $80 \%$ solution in toluene; $15 \mathrm{mmol}$ ) in toluene (30 $\mathrm{mL}$ ) were added tetrabutylammonium bromide $(0.161 \mathrm{~g}, 0.5 \mathrm{mmol})$ and $50 \%$ aqueous $\mathrm{NaOH}(6 \mathrm{~mL})$. The two-phase system was stirred vigorously for $3 \mathrm{~h}$ at room temperature, then it was diluted with toluene $(10 \mathrm{~mL})$ and the phases were separated. The organic layer was washed several times with water and then with brine. It was dried over $\mathrm{Na}_{2} \mathrm{SO}_{4}$ and the solvent was removed under reduced pressure to give the product (2.179 g, 93\%) as an almost colorless oil which darkened slowly on storage. IR (KBr): 3290, 2125, 1564, 1507, 1465, 1189, 1053, 898, $752 \mathrm{~cm}^{-1}$; MS (EI, $\left.70 \mathrm{eV}\right) \mathrm{m} / \mathrm{z}: 235\left(\mathrm{M}^{+}, 46 \%\right), 233\left(\mathrm{M}^{+}\right.$, 49), 196 (12), 194 (10), 154 (100), 127 (18), 115 (33), 88 (22), 62 (19); ${ }^{1} \mathrm{H}-\mathrm{NMR}\left(\mathrm{CDCl}_{3}\right)$ 8: 7.77 (d, $\left.J_{4-6}=1.8 \mathrm{~Hz}, 1 \mathrm{H}, 4-\mathrm{H}\right), 7.36-7.26(\mathrm{~m}, 2 \mathrm{H}, 6-\mathrm{H}, 7-\mathrm{H}), 7.21\left(\mathrm{~d}, J_{2-3}=3.3 \mathrm{~Hz}, 2-\mathrm{H}\right), 6.48\left(\mathrm{~d}, J_{2-3}=3.3\right.$ $\mathrm{Hz}, 1 \mathrm{H}, 3-\mathrm{H}$ ), 4.86 (d, $J=2.6 \mathrm{~Hz}, 2 \mathrm{H}, \mathrm{CH}_{2}$ ), 2.42 (t, $J=2.6 \mathrm{~Hz}, 1 \mathrm{H}, \mathrm{C} \equiv \mathrm{CH}$ ). HRMS (EI, $\left.70 \mathrm{eV}\right) \mathrm{m} / \mathrm{z}$ calcd. for $\mathrm{C}_{11} \mathrm{H}_{8} \mathrm{BrN}\left(\mathrm{M}^{+}\right)$: 232.9840. Found: 232.9844 .

1-Iodophthalazine (2a) [11]. Modified Procedure. A mixture of 1-chlorophthalazine (2.75 g, 17 $\mathrm{mmol})$, potassium iodide $(5.0 \mathrm{~g}, 30 \mathrm{mmol}), 57 \%$ hydroiodic acid $(3.4 \mathrm{~mL})$ and acetone $(50 \mathrm{~mL})$ was stirred in the dark at room temperature for $96 \mathrm{~h}$. The yellow precipitate $(\mathbf{2 a} \cdot \mathrm{HI})$ was collected by filtration, washed with diethyl ether, and dried in vacuo. The material was then suspended in ice-water and the mixture was stirred for $15 \mathrm{~min}$ and made alkaline with dilute ammonium hydroxide. It was extracted several times with $\mathrm{CH}_{2} \mathrm{Cl}_{2}$ and the combined extracts were repeatedly washed with a solution of sodium thiosulfate $(0.5 \mathrm{~g})$ in $1 \%$ ammonium hydroxide, then with brine. Triethylamine $(2 \mathrm{~mL})$ was added to the $\mathrm{CH}_{2} \mathrm{Cl}_{2}$ extract, then it was dried over $\mathrm{Na}_{2} \mathrm{SO}_{4}$. Evaporation of the volatile components gave 2a (3.153 g, 74\%) as yellow crystals, mp 87-96 ${ }^{\circ} \mathrm{C}$ (lit. [11]: $78{ }^{\circ} \mathrm{C}$ ), which were stored in a deep freezer and which were used for the subsequent steps without further purification.

Synthesis of Compounds 3 by Pd-Catalyzed Cross-Coupling Reaction. General Procedure. To a solution of the aryl halide $\mathbf{2 a}, \mathbf{2 b}, \mathbf{2 c}, \mathbf{2 d}$, or $\mathbf{2 e}$ (2.6 mmol), respectively, and the appropriate alkyne 1a, 1b, 1c, or 1d (3.25 mmol), respectively, in dry THF $(6 \mathrm{~mL})$ were added triethylamine $(1.0 \mathrm{~mL}, 7.2$ mmol), CuI (0.015 g, 3 mol\%) and $\mathrm{Pd}\left(\mathrm{PPh}_{3}\right)_{2} \mathrm{Cl}_{2}$ (0.055 g, $3 \mathrm{~mol} \%$ ), and the mixture was flushed with argon. It was then stirred under an argon atmosphere under the conditions (room temperature or reflux) and for the time listed in Table 1. The insoluble material was filtered off and washed carefully with THF. The combined filtrates were evaporated under reduced pressure and the residue was purified by column chromatography (eluent: ethyl acetate or ethyl acetate/light petroleum).

1-[3-(5-Methoxy-1H-indol-1-yl)prop-1-yn-1-yl]-4-methylphthalazine (3a). Prepared from 5-methoxy1-prop-2-yn-1-yl-1H-indole (1a) and 1-iodo-4-methylphthalazine (2b); yield: 0.408 g (47\%), recrystallization from ethyl acetate/light petroleum gave almost colorless crystals, mp $166-168{ }^{\circ} \mathrm{C}$. IR (KBr): 2921, 2231, 1620, 1484, 1383, 1239, 1153, 1029, 848, 799, 771, $619 \mathrm{~cm}^{-1}$; MS (EI, $\left.70 \mathrm{eV}\right) \mathrm{m} / \mathrm{z}$ : 327 (M+, 19\%), 312 (10), 296 (2), 284 (6), 164 (6), 152 (15), 142 (9), 127 (7), 103 (4), 77 (7), 70 (13), 61 (14), 45 (24), 43 (100); ${ }^{1} \mathrm{H}-\mathrm{NMR}\left(\mathrm{CDCl}_{3}\right) \delta: 8.09$ (d, $J=8.7 \mathrm{~Hz}, 1 \mathrm{H}$, phthalazine 8-H), 8.06 (d, $J=$ $9.3 \mathrm{~Hz}, 1 \mathrm{H}$, phthalazine 5- $\mathrm{H}$, shows positive NOE on irradiation at $3.02 \mathrm{ppm}), 7.92-7.80(\mathrm{~m}, 2 \mathrm{H}$, phthalazine 6-H, 7-H), 7.47 (d, $J_{6-7}=9.0 \mathrm{~Hz}, 1 \mathrm{H}$, indole 7-H), 7.31 (d, $J_{2-3}=3.0 \mathrm{~Hz}, 1 \mathrm{H}$, indole 2-H), $7.15\left(\mathrm{~d}, J_{4-6}=2.1 \mathrm{~Hz}, 1 \mathrm{H}\right.$, indole 4-H), $6.96\left(\mathrm{dd}, J_{6-7}=9.0 \mathrm{~Hz}, J_{4-6}=2.4 \mathrm{~Hz}, 1 \mathrm{H}\right.$, indole 6-H), $6.52(\mathrm{~d}$, 
$J_{2-3}=3.0 \mathrm{~Hz}, 1 \mathrm{H}$, indole 3-H), $5.28\left(\mathrm{~s}, 2 \mathrm{H}, \mathrm{CH}_{2}\right), 3.88\left(\mathrm{~s}, 3 \mathrm{H}, \mathrm{OCH}_{3}\right), 3.02\left(\mathrm{~s}, 3 \mathrm{H}, 4-\mathrm{CH}_{3}\right)$. Anal. calcd. for $\mathrm{C}_{21} \mathrm{H}_{17} \mathrm{~N}_{3} \mathrm{O} \cdot 0.25 \mathrm{H}_{2} \mathrm{O}$ : C, 76.00; H, 5.31; N, 12.60. Found: C, 76.04; H, 5.22; N, 12.45 .

1-[3-(5-Methoxy-1H-indol-1-yl)prop-1-yn-1-yl]-4-phenylphthalazine (3b). Prepared from 5-methoxy1-prop-2-yn-1-yl-1H-indole (1a) and 1-iodo-4-phenylphthalazine (2c); yield: 0.552 g (55\%), recrystallization from ethyl acetate gave almost colorless crystals, mp 150-152 ${ }^{\circ} \mathrm{C}$. IR (KBr): 3057, 2939, 2236, 1619, 1576, 1485, 1386, 1239, 1151, 1028, 702, $653 \mathrm{~cm}^{-1}$; MS (EI, $\left.70 \mathrm{eV}\right) \mathrm{m} / \mathrm{z}: 389\left(\mathrm{M}^{+}\right.$, 100\%), 374 (24), 358 (10), 346 (22), 244 (20), 213 (50), 194 (56), 187 (42), 173 (33), 165 (12), 147 (27), 132 (34), 104 (19), 77 (18), 51 (17), 43 (20); ${ }^{1} \mathrm{H}-\mathrm{NMR}\left(\mathrm{CDCl}_{3}\right)$ 8: 8.19-8.16 (m, 1H, phthalazine 8-H), 8.09-8.05 (m, 1H, phthalazine 5-H, shows positive NOE on irradiation at $7.75 \mathrm{ppm}), 7.88-7.83$ (m, 2H, phthalazine 6-H, 7-H), 7.77-7.74 (m, 2H, phenyl 2-H, 6-H), 7.60-7.56 (m, 3H, phenyl 3-H, 4$\mathrm{H}, 5-\mathrm{H}$, shows positive NOE on irradiation at $7.75 \mathrm{ppm}$ ), 7.49 (d, $J_{6-7}=8.9 \mathrm{~Hz}, 1 \mathrm{H}$, indole 7-H), 7.33 $\left(\mathrm{d}, J_{2-3}=3.3 \mathrm{~Hz}, 1 \mathrm{H}\right.$, indole 2-H), $7.16\left(\mathrm{~d}, J_{4-6}=2.4 \mathrm{~Hz}, 1 \mathrm{H}\right.$, indole 4-H), $6.97\left(\mathrm{dd}, J_{6-7}=8.9 \mathrm{~Hz}, J_{4-6}\right.$ $=2.4 \mathrm{~Hz}, 1 \mathrm{H}$, indole 6-H), $6.54\left(\mathrm{~d}, J_{2-3}=3.3 \mathrm{~Hz}, 1 \mathrm{H}\right.$, indole $\left.3-\mathrm{H}\right), 5.31\left(\mathrm{~s}, 2 \mathrm{H}, \mathrm{CH}_{2}\right), 3.88(\mathrm{~s}, 3 \mathrm{H}$, $\mathrm{OCH}_{3}$ ). Anal. calcd. for $\mathrm{C}_{26} \mathrm{H}_{19} \mathrm{~N}_{3} \mathrm{O}$ : C, 80.19; H, 4.92; N, 10.79. Found: C, 79.98; H, 4.99; N, 10.55.

4-[3-(5-Methoxy-1H-indol-1-yl)prop-1-yn-1-yl]isoquinoline (3c). Prepared from 5-methoxy-1-prop-2yn-1-yl-1H-indole (1a) and 4-bromoisoquinoline (2e); yield: $0.284 \mathrm{~g}$ (34\%), recrystallization from ethyl acetate/light petroleum gave brownish crystals, mp 131-133 ${ }^{\circ} \mathrm{C}$. IR (KBr): 2903, 2825, 2244, 1620, 1488, 1241, 1150, 794, 753, 715, $580 \mathrm{~cm}^{-1}$; MS (EI, $\left.70 \mathrm{eV}\right) \mathrm{m} / \mathrm{z}: 312\left(\mathrm{M}^{+}, 60 \%\right), 296$ (2), 281 (3), 201 (4), 166 (100), 156 (9), 139 (36), 103 (5), 89 (6), 76 (6), 43 (14); ${ }^{1} \mathrm{H}$ NMR (CDCl $)$ ) 9.28 (br $\mathrm{s}, 1 \mathrm{H}$, isoquinoline $1-\mathrm{H}), 8.75(\mathrm{br} \mathrm{s}, 1 \mathrm{H}$, isoquinoline $3-\mathrm{H}), 8.11\left(\mathrm{~d}, J_{7-8}=8.4 \mathrm{~Hz}, 1 \mathrm{H}\right.$, isoquinoline 8$\mathrm{H}), 7.98\left(\mathrm{~d}, J_{5-6}=7.8 \mathrm{~Hz}, 1 \mathrm{H}\right.$, isoquinoline $\left.5-\mathrm{H}\right), 7.74(\mathrm{t}, J=7.7 \mathrm{~Hz}, 1 \mathrm{H}$, isoquinoline 7-H, shows positive NOE on irradiation at $8.11 \mathrm{ppm}), 7.65\left(\mathrm{t}, J=7.3 \mathrm{~Hz}, 1 \mathrm{H}\right.$, isoquinoline 6-H), 7.46 (d, $J_{6-7}=8.9$ $\mathrm{Hz}, 1 \mathrm{H}$, indole $7-\mathrm{H}$, shows positive NOE on irradiation at $5.25 \mathrm{ppm}), 7.32\left(\mathrm{~d}, J_{2-3}=3.3 \mathrm{~Hz}, 1 \mathrm{H}\right.$, indole 2-H, shows positive NOE on irradiation at $5.25 \mathrm{ppm}$ ), 7.15 (d, $J_{4-6}=2.4 \mathrm{~Hz}, 1 \mathrm{H}$, indole 4-H), 6.96 (dd, $J_{6-7}=8.9 \mathrm{~Hz}, J_{4-6}=2.4 \mathrm{~Hz}, 1 \mathrm{H}$, indole 6-H), 6.52 (d, $J_{2-3}=3.3 \mathrm{~Hz}, 1 \mathrm{H}$, indole 3-H), $5.25\left(\mathrm{~s}, 2 \mathrm{H}, \mathrm{CH}_{2}\right.$ ), 3.88 (s, 3H, $\mathrm{OCH}_{3}$ ). Anal. calcd for $\mathrm{C}_{21} \mathrm{H}_{16} \mathrm{~N}_{2} \mathrm{O} \cdot 0.25 \mathrm{H}_{2} \mathrm{O}: \mathrm{C}, 79.60 ; \mathrm{H}, 5.25 ; \mathrm{N}, 8.84$. Found: C, 79.66; H, 5.50; N, 8.46.

1-[3-(5-Methoxy-1H-indol-1-yl)prop-1-yn-1-yl]isoquinoline (3d). Prepared from 5-methoxy-1-prop-2yn-1-yl-1H-indole (1a) and 1-iodoisoquinoline (2d); yield: $0.620 \mathrm{~g}$ (75\%), recrystallization from ethyl acetate/light petroleum gave almost colorless crystals, mp 155-157 ${ }^{\circ} \mathrm{C}$. IR (KBr): 3048, 2953, 2235, 1620, 1578, 1551, 1486, 1350, 1242, 1153, 1028, 830, 747, 723, $666 \mathrm{~cm}^{-1}$; MS (EI, $\left.70 \mathrm{eV}\right) \mathrm{m} / \mathrm{z}: 312$ (M+, 34\%), 297 (6), 281 (2), 269 (9), 184 (11), 166 (44), 146 (11), 139 (38), 134 (15), 103 (7), 89 (6), 76 (9), 61 (13), 45 (26), 43 (100); ${ }^{1} \mathrm{H}-\mathrm{NMR}\left(\mathrm{CDCl}_{3}\right) \delta: 8.51$ (d, $J_{3-4}=5.4 \mathrm{~Hz}, 1 \mathrm{H}$, isoquinoline 3-H), $8.22\left(\mathrm{dd}, J_{7-8}=8.4 \mathrm{~Hz}, J_{6-8}=1.2 \mathrm{~Hz}, 1 \mathrm{H}\right.$, isoquinoline 8-H), 7.82 (d, $J_{5-6}=8.1 \mathrm{~Hz}, 1 \mathrm{H}$, isoquinoline 5$\mathrm{H}), 7.72-7.69$ ( $\mathrm{m}, 1 \mathrm{H}$, isoquinoline $6-\mathrm{H}$, shows positive NOE on irradiation at 7.82 ppm), 7.67-7.63 (m, $1 \mathrm{H}$, isoquinoline 4- $\mathrm{H}$, shows positive NOE on irradiation at $8.51 \mathrm{ppm}), 7.61-7.55(\mathrm{~m}, 1 \mathrm{H}$, isoquinoline 7- $\mathrm{H}$, shows positive NOE on irradiation at $8.22 \mathrm{ppm}$ ), 7.47 (d, $J_{6-7}=8.9 \mathrm{~Hz}, 1 \mathrm{H}$, indole 7$\mathrm{H}), 7.32\left(\mathrm{~d}, J_{2-3}=3.3 \mathrm{~Hz}, 1 \mathrm{H}\right.$, indole 2-H), 7.14 (d, $J_{4-6}=2.4 \mathrm{~Hz}, 1 \mathrm{H}$, indole 4-H), 6.96 (dd, $J_{6-7}=8.9$ $\mathrm{Hz}, J_{4-6}=2.4 \mathrm{~Hz}, 1 \mathrm{H}$, indole 6-H), $6.51\left(\mathrm{~d}, J_{2-3}=3.3 \mathrm{~Hz}, 1 \mathrm{H}\right.$, indole 3-H), $5.26\left(\mathrm{~s}, 2 \mathrm{H}, \mathrm{CH}_{2}\right), 3.88$ (s, 
3H, $\mathrm{OCH}_{3}$ ). Anal. calcd. for $\mathrm{C}_{21} \mathrm{H}_{16} \mathrm{~N}_{2} \mathrm{O} \cdot 0.2 \mathrm{H}_{2} \mathrm{O}$ : C, 79.83; H, 5.23; N, 8.87. Found: C, 79.82; $\mathrm{H}$, 5.22; N, 8.59.

1-[3-(2,3-Dihydro-1H-indol-1-yl)prop-1-yn-1-yl]phthalazine (3e). Preparation from 1-prop-2-yn-1ylindoline (1d) and 1-iodophthalazine (2a); yield: $0.592 \mathrm{~g}$ (80\%), recrystallization from ethyl acetate gave brownish crystals, mp 94-95 ${ }^{\circ} \mathrm{C}$. IR (KBr): 3043, 2830, 2227, 1606, 1484, 1353, 1237, 1138, 757, $594 \mathrm{~cm}^{-1}$; MS (EI, $70 \mathrm{eV}$ ) m/z: 285 (M+2\%), 168 (100), 141 (13), 114 (11), 91 (23), 77 (6), 65 (16), 51 (5); ${ }^{1} \mathrm{H}-\mathrm{NMR}\left(\mathrm{CDCl}_{3}\right) \delta$ : 9.43 (s, $1 \mathrm{H}$, phthalazine 4-H), 7.93-7.73 (m, 4H, phthalazine 5-H, 6$\mathrm{H}, 7-\mathrm{H}, 8-\mathrm{H}), 7.21-7.16$ (m, 2H, indoline 4-H, 6-H, shows positive NOE on irradiation at $3.05 \mathrm{ppm}$ ), 6.85-6.76 (m, 2H, indoline 5- $\mathrm{H}$, indoline 7-H), $4.38\left(\mathrm{~s}, 2 \mathrm{H}, \mathrm{C} \equiv \mathrm{CCH}_{2}\right), 3.60(\mathrm{t}, J=8.1 \mathrm{~Hz}, 2 \mathrm{H}$, indoline 2-H, shows positive NOE on irradiation at $3.05 \mathrm{ppm}$ ), 3.05 (t, $J=8.1 \mathrm{~Hz}, 2 \mathrm{H}$, indoline 3-H). Anal. calcd. for $\mathrm{C}_{19} \mathrm{H}_{15} \mathrm{~N}_{3}$ : C, 79.98; H, 5.30; N, 14.73. Found: C, 79.93; H, 5.47; N, 14.68.

1-[3-(5-Bromo-1H-indol-1-yl)prop-1-yn-1-yl]phthalazine (3f). Preparation from 5-bromo-1-prop-2-yn1-yl-1H-indole (1b) and 1-iodophthalazine (2a); yield: $0.281 \mathrm{~g}$ (30\%), recrystallization from ethyl acetate gave almost colorless crystals, mp 154-158 ${ }^{\circ} \mathrm{C}$. IR (KBr): 3097, 2955, 2239, 1465, 1394, 1353, 1282, 1185, 1051, 899, 758, $594 \mathrm{~cm}^{-1}$; MS (EI, $\left.70 \mathrm{eV}\right) \mathrm{m} / \mathrm{z}: 363$ (M+13\%), $361\left(\mathrm{M}^{+}, 12\right), 282$ (8), 195 (14), 167 (7), 141 (34), 127 (36), 114 (15), 97 (11), 84 (20), 69 (25), 58 (93), 57 (39), 43 (100); ${ }^{1} \mathrm{H}$ NMR $\left(\mathrm{CDCl}_{3}\right) \delta: 9.48\left(\mathrm{~s}, 1 \mathrm{H}\right.$, phthalazine 4-H), 8.06 (d, $J_{7-8}=8.1 \mathrm{~Hz}, 1 \mathrm{H}$, phthalazine 8-H), 7.99-7.86 (m, 3H, phthalazine 5-H, 6-H, 7-H), $7.81\left(\mathrm{~d}, J_{4-6}=1.6 \mathrm{~Hz}, 1 \mathrm{H}\right.$, indole 4-H), 7.45 (d, $J_{6-7}=8.7 \mathrm{~Hz}, 1 \mathrm{H}$, indole 7-H), 7.38 (dd, $J_{6-7}=8.7 \mathrm{~Hz}, J_{4-6}=1.6 \mathrm{~Hz}, 1 \mathrm{H}$, indole 6-H), 7.34 (d, $J_{2-3}=3.3 \mathrm{~Hz}, 1 \mathrm{H}$, indole 2-H), 6.55 (d, $J_{2-3}=3.3 \mathrm{~Hz}, 1 \mathrm{H}$, indole 3-H), 5.31 (s, $2 \mathrm{H}, \mathrm{CH}_{2}$ ). Anal. calcd. for $\mathrm{C}_{19} \mathrm{H}_{12} \mathrm{~N}_{3} \mathrm{Br}$ : C, 63.00; H, 3.34; N, 11.60. Found: C, 62.75; H, 3.34; N, 11.34 .

Methyl 1-(3-Phthalazin-1-ylprop-2-yn-1-yl)-1H-indole-5-carboxylate (3g). Preparation from methyl 1prop-2-yn-1-yl-1H-indole-5-carboxylate (1c) and 1-iodophthalazine (2a); modified work-up procedure: after completion of the reaction, the solid material ( $\mathbf{g} \mathbf{g}+$ triethylammonium iodide) was filtered off and washed with diethyl ether. It was then dissolved in $\mathrm{CH}_{2} \mathrm{Cl}_{2}$ and this solution was washed with $0.5 \mathrm{~N} \mathrm{HCl}$, then with water. The extract was dried over $\mathrm{Na}_{2} \mathrm{SO}_{4}$ and evaporated under reduced pressure to give the product $(0.133 \mathrm{~g}, 15 \%)$ as brownish crystals, mp $97^{\circ} \mathrm{C}$ (decomposition). IR (KBr): 2948, 2241, 1710, 1610, 1434, 1310, 1196, 1097, 754, $595 \mathrm{~cm}^{-1}$; MS (EI, $\left.70 \mathrm{eV}\right) \mathrm{m} / \mathrm{z}: 341$ (M+2 23\%), 282 (10), 175 (50), 165 (10), 144 (100), 141 (36), 116 (73), 89 (39), 72 (29), 58 (66), 44 (81); ${ }^{1} \mathrm{H}-\mathrm{NMR}\left(\mathrm{CDCl}_{3}\right) \delta$ : 9.49 (s, $1 \mathrm{H}$, phthalazine 4-H), 8.45 (d, $J_{4-6}=0.9 \mathrm{~Hz}, 1 \mathrm{H}$, indole 4-H), 8.107.85 (m, 5H, indole 6-H, phthalazine 5-H, 6-H, 7-H, 8-H), 7.58 (d, $J_{6-7}=8.7 \mathrm{~Hz}, 1 \mathrm{H}$, indole 7-H, shows positive NOE on irradiation at $5.35 \mathrm{ppm}$ ), $7.41\left(\mathrm{~d}, J_{2-3}=3.4 \mathrm{~Hz}, 1 \mathrm{H}\right.$, indole $2-\mathrm{H}$, shows positive NOE on irradiation at $5.35 \mathrm{ppm}$ ), 6.71 (d, $J_{2-3}=3.4 \mathrm{~Hz}, 1 \mathrm{H}$, indole 3-H), 5.35 (s, 2H, $\mathrm{CH}_{2}$ ), 3.95 (s, $3 \mathrm{H}, \mathrm{OCH}_{3}$ ). HRMS (EI, $70 \mathrm{eV}$ ) $\mathrm{m} / \mathrm{z}$ calcd. for $\mathrm{C}_{21} \mathrm{H}_{15} \mathrm{~N}_{3} \mathrm{O}_{2}\left(\mathrm{M}^{+}\right)$: 341.1164. Found: 341.1167.

\section{Acknowledgements}

We are grateful to Æterna Zentaris GmbH, Frankfurt/Main (Germany) for the in-vitro evaluation of antitumor activity. 


\section{References and Notes}

1. Haider, N.; Jbara, R.; Khadami, F.; Wanko, R. Synthesis of Pyridazino[4,5-b]carbazoles as Potential Antineoplastic Agents. Heterocycles 1998, 48, 1609-1622.

2. Haider, N.; Käferböck, J.; Mátyus, P. Diels-Alder Reaction of Pyrano[3,4-b]indolones with an Electron-Deficient Pyridazinone: a New Pathway to Carbazole-Fused Pyridazines. Heterocycles 1999, 51, 2703-2710.

3. Fidesser, E.; Haider, N.; Jbara, R. Convenient Synthesis of New 3-Aminocarbazole and Pyrimido[5,4-b]carbazole Derivatives. ARKIVOC 2001, 2, 133-139.

4. Haider, N. Pyridazine-Fused Carbazoles: Synthesis, Reactivity, and Antitumor Activity. J. Heterocycl. Chem. 2002, 39, 511-521.

5. Haider, N.; Sotelo, E. 1,5-Dimethyl-6H-pyridazino[4,5-b]carbazole, a 3-Aza Bioisoster of the Antitumor Alkaloid Olivacine. Chem. Pharm. Bull. 2002, 50, 1479-1483.

6. Shanab, K.; Pongprom, N.; Wulz, E.; Holzer, W.; Spreitzer, H.; Schmidt, P.; Aicher, B.; Müller, G.; Günther, E. Synthesis and Biological Evaluation of Novel Cytotoxic Azanaphthoquinone Annelated Pyrrolo Oximes. Bioorg. Med. Chem. Lett. 2007, in press.

7. Spreitzer, H.; Pichler, A.; Holzer, W.; Kratzel, M.; Slanz, R.; Koulouri, A.; Krenn, P.; Parrer, U.; Szieber, P. Synthesis of azanaphthoquinone annelated pyrroles. Heterocycles 2001, 54, 111-121.

8. Spreitzer, H.; Puschmann, C. Synthesis of Anticancer Compounds, I, "Dual Function" Antitumor Agents Based on Bioreduction and DNA-Alkylation. Monatsh. Chem. 2007, 138, 517-522.

9. Haider, N.; Käferböck, J. Intramolecular [4+2] cycloaddition reactions of indolylalkylpyridazines: synthesis of annulated carbazoles. Tetrahedron 2004, 60, 6495-6507.

10. Broggini, G.; Bruché, L.; Zecchi, G.; Pilati, T. 1,3-Dipolar cycloadditions to nitrogen-substituted allenes. J. Chem. Soc., Perkin Trans. 1 1990, 533-539.

11. Hirsch, A.; Orphanos, D. G. The synthesis of some iodophthalazines and phthalazinecarbonitriles. Can. J. Chem. 1966, 44, 1551-1554.

12. Gabriel, S.; Eschenbach, G. Über eine Darstellungsweise der Phthalazine. Chem. Ber. 1897, 30, 3022-3037.

13. Lieck, A. Über einige Phthalazine. Chem. Ber. 1905, 38, 3918-3924.

14. Hayashi, E.; Akahori, Y.; Yamamoto, Y. 1-Nitroisoquinoline. Yakugaku Zasshi 1967, 87, 13421345; [Chem. Abstr. 1968, 69, 2847].

15. Ukai, T. Mercuric derivatives of quinoline, methylquinoline and isoquinoline. VII. (Supplement). Yakugaku Zasshi 1931, 51, 542-576; [Chem. Abstr. 1931, 25, 48018].

16. Haider, N.; Kabicher, T. Methyl 1-prop-2-yn-1-yl-1H-indole-5-carboxylate. MolBank 2007, accepted.

17. Torregrosa, J. L.; Baboulene, M.; Speziale, V.; Lattes, A. Hydroboration of unsaturated amines. V. New approach to aminoalkylidenecycloalkanes. J. Organomet. Chem. 1983, 244, 311-317.

18. For recent examples of alkyne/allene rearrangements of $N$-propargylindoles, see: a) lit. [9]; b) Abbiati, G.; Canevari, V.; Caimi, S.; Rossi, E. Domino addition/annulation of delta-alkynylaldehydes and oxygen nucleophiles. A new entry to [1,4]oxazino[4,3-a]indoles. Tetrahedron Lett. 2005, 46, 7117-7120. 
19. Scudiero, D. A.; Shoemaker, R. H.; Paull, K. D.; Monks, A.; Tierney, S.; Nofziger, T. H.; Currens, M. J.; Seniff, D.; Boyd, M. R. Evaluation of a soluble tetrazolium/formazan assay for cell growth and drug sensitivity in culture using human and other tumor cell lines. Cancer Res. 1988, 48, 4827-4833.

Sample Availability: available from the authors.

(C) 2007 by MDPI (http://www.mdpi.org). Reproduction is permitted for noncommercial purposes. 